\title{
ELEMENTS OF THE KKM THEORY ON ABSTRACT CONVEX SPACES
}

\author{
SEHIE PARK
}

\begin{abstract}
We introduce a new concept of abstract convex spaces and a multimap class $\mathfrak{K}$ having certain KKM property. From a basic KKM type theorem for a $\mathfrak{K}$-map defined on an abstract convex space without any topology, we deduce ten equivalent formulations of the theorem. As applications of the equivalents, in the frame of abstract convex topological spaces, we obtain Fan-Browder type fixed point theorems, almost fixed point theorems for multimaps, mutual relations between the map classes $\mathfrak{K}$ and $\mathfrak{B}$, variational inequalities, the von Neumann type minimax theorems, and the Nash equilibrium theorems.
\end{abstract}

\section{Introduction}

Many problems in nonlinear analysis can be solved by showing the nonemptyness of the intersection of certain family of subsets of an underlying set. Each point of the intersection can be a fixed point, a coincidence point, an equilibrium point, a saddle point, an optimal point, or others of the corresponding equilibrium problem under consideration. The first remarkable result on the nonempty intersection was the celebrated Knaster-Kuratowski-Mazurkiewicz theorem (simply, the KKM principle) in 1929 [25], which concerns with certain types of multimaps called the KKM maps later.

The KKM theory, first called by the author in [30, 31], was the study of KKM maps and their applications. Nowadays, it would be better to regard it as the study of applications of various equivalent formulations of the KKM principle and their generalizations. At the beginning, the theory was mainly devoted to study on convex subsets of topological vector spaces. Later, it has been extended to convex spaces by Lassonde [26], and to $C$-spaces (or $H$-spaces) by Horvath [19-22] and others. In the last decade, the KKM theory is extended to generalized convex ( $G$-convex) spaces in a sequence of papers of the author; for details, see [35-42,44-47] and references therein. It is noted that, in the

Received June 12, 2006.

2000 Mathematics Subject Classification. Primary 47H04, 47H10; Secondary 46A16, 46A55, 52A07, 54C60, 54H25, 55M20.

Key words and phrases. abstract convex space, generalized convex space, KKM principle, multimap (map) classes $\mathfrak{K}, \mathfrak{K C}, \mathfrak{K O}$, coincidence, almost fixed point, map classes $\mathfrak{A}_{c}^{\kappa}, \mathfrak{B}$. 
KKM theory, there have appeared a number of coincidence theorems with many significant applications.

Recently, in [41], we introduced a new concept of abstract convex spaces and certain broad classes $\mathfrak{K C}$ and $\mathfrak{K O}$ of multimaps (having the KKM property). With this new concept, the KKM type maps were used to obtain coincidence theorems and fixed point theorems.

In the first half of this paper, we recall the concept of abstract convex spaces and show that they are adequate to establish the KKM theory. From a KKM type theorem for a $\mathfrak{K}$-map on an abstract convex space, we deduce ten equivalent formulations of the theorem in the forms of the Ky Fan type matching theorem, a coincidence theorem, a finite intersection theorem for multimap values, analytic alternatives, minimax inequalities, and geometric or section properties. These are all known to be the basic elements of the KKM theory on $G$-convex spaces, $H$-spaces, and convex spaces. Therefore, we show that the basic results in the KKM theory can be stated for abstract convex spaces without any topology.

In the second half, we are mainly concerned with applications of the equivalents of the coincidence theorem for particular abstract convex topological spaces. Actually in the frame of such spaces, we obtain Fan-Browder type fixed point theorems, almost fixed point theorems for multimaps, mutual relations between the map classes $\mathfrak{K}$ and $\mathfrak{B}$, variational inequalities, the von Neumann type minimax theorems, and the Nash equilibrium theorems.

Consequently, in the present paper, we generalize and unify most of important results in the elements or foundations of the KKM theory appeared in our previous works [30-33, 36, 40-42, 45-47]. In fact, the generalized convex space theory established in these works is now extended to abstract convex topological spaces satisfying the KKM principle.

\section{Abstract convex spaces and the map class $\mathfrak{K}$}

In this section, we recall the definitions in [41] and new examples.

Let $\langle D\rangle$ denote the set of all nonempty finite subsets of a set $D$.

Definitions. An abstract convex space $(E, D ; \Gamma)$ consists of a nonempty set $E$, a nonempty set $D$, and a multimap $\Gamma:\langle D\rangle \multimap E$ with nonempty values. We sometimes denote $\Gamma_{A}:=\Gamma(A)$ for $A \in\langle D\rangle$.

Let $(E, D ; \Gamma)$ be an abstract convex space. For any $D^{\prime} \subset D$, the $\Gamma$-convex hull of $D^{\prime}$ is denoted and defined by

$$
\operatorname{co}_{\Gamma} D^{\prime}:=\bigcup\left\{\Gamma_{A} \mid A \in\left\langle D^{\prime}\right\rangle\right\} \subset E .
$$

[co is reserved for the convex hull in vector spaces.] A subset $X$ of $E$ is called a $\Gamma$-convex subset of $(E, D ; \Gamma)$ relative to $D^{\prime}$ if for any $N \in\left\langle D^{\prime}\right\rangle$, we have $\Gamma_{N} \subset X$, that is, $\operatorname{co}_{\Gamma} D^{\prime} \subset X$. 
When $D \subset E$, the space is denoted by $(E \supset D ; \Gamma)$. In such case, a subset $X$ of $E$ is said to be $\Gamma$-convex if $\operatorname{co}_{\Gamma}(X \cap D) \subset X$; in other words, $X$ is $\Gamma$-convex relative to $D^{\prime}:=X \cap D$. In case $E=D$, let $(E ; \Gamma):=(E, E ; \Gamma)$.

An abstract convex space with any topology is called an abstract convex topological space.

Examples. 1. A convexity space $(E, \mathcal{C})$ in the classical sense consists of a nonempty set $E$ and a family $\mathcal{C}$ of subsets of $E$ such that $E$ itself is an element of $\mathcal{C}$ and $\mathcal{C}$ is closed under arbitrary intersection. For details, see [49], where the bibliography lists 283 papers. For any subset $X \subset E$, its $\mathcal{C}$-convex hull is defined and denoted by $\operatorname{Co}_{\mathcal{C}} X:=\bigcap\{Y \in \mathcal{C} \mid X \subset Y\}$. We say that $X$ is $\mathcal{C}$-convex if $X=\operatorname{Co}_{\mathcal{C}} X$. Now we can consider the map $\Gamma:\langle E\rangle \multimap E$ given by $\Gamma_{A}:=\operatorname{Co}_{\mathcal{C}} A$ for each $A \in\langle E\rangle$. Then $(E, \mathcal{C})$ becomes our abstract convex space $(E ; \Gamma)$.

2. A generalized convex space or a $G$-convex space $(X, D ; \Gamma)$ consists of a topological space $X$, a nonempty set $D$, and a multimap $\Gamma:\langle D\rangle \rightarrow X$ such that for each $A \in\langle D\rangle$ with the cardinality $|A|=n+1$, there exists a continuous function $\phi_{A}: \Delta_{n} \rightarrow \Gamma(A)$ such that $J \in\langle A\rangle$ implies $\phi_{A}\left(\Delta_{J}\right) \subset \Gamma(J)$.

Here, $\Delta_{n}$ is the standard $n$-simplex with vertices $\left\{e_{i}\right\}_{i=0}^{n}$, and $\Delta_{J}$ the face of $\Delta_{n}$ corresponding to $J \in\langle A\rangle$; that is, if $A=\left\{a_{0}, a_{1}, \ldots, a_{n}\right\}$ and $J=$ $\left\{a_{i_{0}}, a_{i_{1}}, \ldots, a_{i_{k}}\right\} \subset A$, then $\Delta_{J}=\operatorname{co}\left\{e_{i_{0}}, e_{i_{1}}, \ldots, e_{i_{k}}\right\}$.

For details on $G$-convex spaces, see $[35-42,44-47]$, where basic theory was extensively developed and lots of examples of $G$-convex spaces were given.

3. A well-known subclass of $G$-convex spaces due to Horvath [19-22] can be generalized as follows: A $G$-convex space $(X, D ; \Gamma)$ is called an $H$-space if each $\Gamma_{A}$ is $\omega$-connected (that is, $n$-connected for all $n \geq 0$ ) and $\Gamma_{A} \subset \Gamma_{B}$ for $A \subset B$ in $\langle D\rangle$. An $H$-space $(X ; \Gamma)$ is called a $C$-space $[2 \overline{1}]$.

4. If $X$ is a subset of a vector space, $D \subset X$ such that $\operatorname{co} D \subset X$, and each $\Gamma_{A}$ is the convex hull of $A \in\langle D\rangle$ equipped with the Euclidean topology, then $(X, D ; \Gamma)$ becomes a convex space generalizing the one due to Lassonde [26]. Note that any convex subset of a topological vector space (t.v.s.) is a convex space, but not conversely.

5. Let $E$ be a t.v.s. with a neighborhood system $\mathcal{V}$ of its origin. A subset $X$ of $E$ is said to be almost convex [18] if for any $V \in \mathcal{V}$ and for any finite subset $A:=\left\{x_{1}, x_{2}, \ldots, x_{n}\right\}$ of $X$, there exists a subset $B:=\left\{y_{1}, y_{2}, \ldots, y_{n}\right\}$ of $X$ such that $y_{i}-x_{i} \in V$ for each $i=1,2, \ldots, n$ and $\operatorname{co} B \subset X$. By choosing $\Gamma_{A}:=B$ for each $A \in\langle X\rangle,(X ; \Gamma)$ becomes an abstract convex space.

Definitions. Let $(E, D ; \Gamma)$ be an abstract convex space and $Z$ a set. For a multimap $F: E \multimap Z$ with nonempty values, if a multimap $G: D \multimap Z$ satisfies

$$
F\left(\Gamma_{A}\right) \subset G(A):=\bigcup_{y \in A} G(y) \quad \text { for all } A \in\langle D\rangle
$$


then $G$ is called a $K K M$ map with respect to $F$. A $K K M$ map $G: D \multimap E$ is a KKM map with respect to the identity map $1_{E}$.

A multimap $F: E \multimap Z$ is called $a \mathfrak{K}$-map if, for a KKM map $G: D \multimap Z$ with respect to $F$, the family $\{G(y)\}_{y \in D}$ has the finite intersection property. We denote

$$
\mathfrak{K}(E, Z):=\{F: E \multimap Z \mid F \text { is a } \mathfrak{K} \text {-map }\} .
$$

Similarly, when $Z$ is a topological space, a $\mathfrak{K C}$-map is defined for closedvalued maps $G$, and a $\mathfrak{K O}$-map for open-valued maps $G$. Note that if $Z$ is discrete then three classes $\mathfrak{K}, \mathfrak{K C}$, and $\mathfrak{K O}$ are identical. Some authors use the notation $\operatorname{KKM}(E, Z)$ instead of $\mathfrak{K C}(E, Z)$.

Examples. 1. Every abstract convex space in our sense has a map $F \in$ $\mathfrak{K}(E, Z)$ for any nonempty set $Z$. In fact, for each $x \in E$, choose $F(x):=Z$ or $F(x):=\left\{z_{0}\right\}$ for some $z_{0} \in Z$.

If $1_{E} \in \mathfrak{K}(E, E)$, then $f \in \mathfrak{K}(E, Z)$ for any function $f: E \rightarrow Z$. If $E$ and $Z$ are topological spaces, this holds for $\mathfrak{K C}$ or $\mathfrak{K O}$ for any continuous $f$.

2. For a $G$-convex space $(X, D ; \Gamma)$ and a topological space $Z$, we defined the classes $\mathfrak{K}, \mathfrak{K C}, \mathfrak{K O}$ of multimaps $F: X \multimap Z[40]$. It is known that for a $G$ convex space $(X, D ; \Gamma)$, we have the identity map $1_{X} \in \mathfrak{K C}(X, X) \cap \mathfrak{K O}(X, X)$; see [24, 36, 40]. Moreover, if $F: X \rightarrow Z$ is a continuous single-valued map or if $F: X \rightarrow Z$ has a continuous selection, then it is easy to check that $F \in \mathfrak{K C}(X, Z) \cap \mathfrak{K O}(X, Z)$. Note that there are many known selection theorems due to Michael and others.

3. In early 1990 's, the author introduced the admissible class $\mathfrak{A}_{c}^{\kappa}(X, Y)$ of multimaps $X \multimap Y$ between topological spaces and showed that $\mathfrak{A}_{c}^{\kappa}(X, Y) \subset$ $\mathfrak{K C}(X, Y)$ when $X$ is a convex space and $Y$ is a Hausdorff space. Motivated by this, Chang and Yen [9] defined the KKM class of maps on convex subsets of topological vector spaces. Moreover, Chang et al. [10] extended the KKM-class to $S$-KKM class. On the other hand, the author extended the $\mathfrak{A}_{c}^{\kappa}$-class to the 'better' admissible $\mathfrak{B}$-class on convex spaces, supplied a large number of examples, and showed that, in the class of compact closed multimaps from convex spaces into Hausdorff spaces, two subclasses $\mathfrak{B}$ and $\mathfrak{K C}$ coincide [33]. Recently H. Kim [23] showed that two classes KKM and $s$-KKM of multimaps from a convex space into a topological space are identical whenever $s$ is surjective [this is the only case $s$-KKM is meaningful]. For $G$-convex spaces, such multimap classes are extended and investigated by a number of authors.

4. Modifying the original definition of S-KKM maps of Chang et al. [10], Amini et al. [4] defined the S-KKM class for a classical convexity space $(X, \mathcal{C})$ with a nonempty set $Z$ and a topological space $Y$ as follows: If $S: Z \multimap X$, $F: X \multimap Y$, and $G: Z \multimap Y$ are three multimaps satisfying

$$
F\left(\operatorname{Co}_{\mathcal{C}}(S(A)) \subset G(A) \text { for each } A \in\langle Z\rangle,\right.
$$


then $G$ is called a $\mathcal{C}$-S-KKM map with respect to $F$. If the map $F: X \multimap Y$ satisfies the requirement that for any $\mathcal{C}-\mathrm{S}-\mathrm{KKM}$ map $G$ with respect to $F$, the family $\{\overline{G(z)} \mid z \in Z\}$ has the finite intersection property, then $F$ is said to have the S-KKM property with respect to $\mathcal{C}$. Amini et al. defined

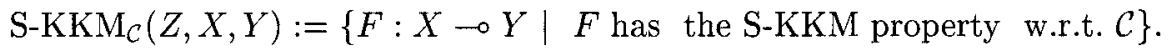

It should be noticed that, by putting $\Gamma_{A}:=\operatorname{Co}(S(A))$ for each $A \in\langle Z\rangle$, the triple $(X, Z ; \Gamma)$ becomes an abstract convex space in our sense and $\mathrm{S}$ $\operatorname{KKM}_{\mathcal{C}}(Z, X, Y)$ becomes simply $\mathfrak{K C}(X, Y)$. Similarly, we can define $\mathfrak{K O}(X, Y)$ and $\mathfrak{K}(X, Y)$ even if $Y$ is a mere set.

We can define a subspace of an abstract convex space and $\mathfrak{K}$-maps on subspaces. The following generalize [41, Propositions 1 and 2], resp., with slightly modified proofs:

Lemma 1. For an abstract convex space $(E, D ; \Gamma)$ and a nonempty subset $D^{\prime}$ of $D$, let $X$ be a $\Gamma$-convex subset of $E$ relative to $D^{\prime}$ and $\Gamma^{\prime}:\left\langle D^{\prime}\right\rangle \multimap X$ a map defined by

$$
\Gamma_{A}^{\prime}:=\Gamma_{A} \subset X \text { for } A \in\left\langle D^{\prime}\right\rangle
$$

Then $\left(X, D^{\prime} ; \Gamma^{\prime}\right)$ itself is an abstract convex space called a subspace.

Lemma 2. Let $(E, D ; \Gamma)$ be an abstract convex space, $\left(X, D^{\prime} ; \Gamma^{\prime}\right)$ a subspace, and $Z$ a set. If $F \in \mathfrak{K}(E, Z)$, then $\left.F\right|_{X} \in \mathfrak{K}(X, Z)$.

Note that Lemma 2 holds for $\mathfrak{K C}$ and $\mathfrak{K O}$ instead of $\mathfrak{K}$ whenever $Z$ is a topological space.

From now on, multimaps are simply called maps and sometimes we will not distinguish a map with its graph.

\section{The KKM type theorems}

From the definitions of each of the classes $\mathfrak{K}, \mathfrak{K C}$ and $\mathfrak{K O}$, we can deduce ten equivalent statements. For simplicity, we will do only for the class $\mathfrak{K}$.

First of all, as in [41], we obtain the following prototype of KKM type theorems for abstract convex spaces:

Theorem 1. Let $(E, D ; \Gamma)$ be an abstract convex space, $Z$ a set, and $F: E \multimap$ $Z$ a map. Then $F \in \mathfrak{K}(E, Z)$ if and only if for any map $G: D \multimap Z$ satisfying

(1.1) $F\left(\Gamma_{N}\right) \subset G(N)$ for any $N \in\langle D\rangle$, we have $F(E) \cap \bigcap\{G(y) \mid y \in N\} \neq \emptyset$ for each $N \in\langle D\rangle$.

Proof. For the necessity, from (1.1), for any $N \in\langle D\rangle$, we have $F\left(\Gamma_{N}\right) \subset F(E) \cap$ $G(N)=\bigcup_{y \in N}\{F(E) \cap G(y)\}$. Since $F$ is a $\mathfrak{K}$-map, the family $\{F(E) \cap G(y)\}_{y \in D}$ has the finite intersection property. The sufficiency is clear.

Remarks. 1. If $Z$ is a topological space and $G$ is open-valued [resp., closedvalued], then we can assume $F \in \mathfrak{K O}(E, Z)$ [resp., $F \in \mathfrak{K C}(E, Z)]$. 
2. If $E=Z=\Delta_{n}$ is an $n$-simplex, $D$ is the set of its vertices, $\Gamma=$ co is the convex hull operation, then the celebrated KKM principle [25] says that $1_{E} \in \mathfrak{K C}(E, E)$. This principle was first used in [25] to obtain one of the most direct proofs of the Brouwer fixed point theorem, and later applied to topological results on Euclidean spaces in $[1,2]$; see $[36,43]$.

3. If $D$ is a nonempty subset of a topological vector space $E=Z$ (not necessarily Hausdorff), Fan's KKM lemma [11] says that $1_{E} \in \mathfrak{K C}(E, E)$. Fan [11] applied his lemma to coincidence theorems generalizing the Tychonoff fixed point theorem and a result concerning two continuous maps from a compact convex set into a uniform space. Later, Fan [12] also applied his lemma to obtain an intersection theorem (concerning sets with convex sections) which implies the Sion minimax theorem [48] and the Tychonoff fixed point theorem.

4. Usually, a KKM theorem is a claim $1_{E} \in \mathfrak{K C}(E, E)$ for an abstract convex topological space $(E, D ; \Gamma)$. For another forms of the KKM theorem for convex spaces, $H$-spaces, or $G$-convex spaces and their applications, there are a large number of works. They can be deduced from Theorem 1 . We give an example as follows:

Corollary 1.1. Let $(E, D ; \Gamma)$ be an abstract convex topological space. Then the identity map $1_{E}$ belongs to $\mathfrak{K C}(E, E)\left[\right.$ resp., $\left.1_{E} \in \mathfrak{K O}(E, E)\right]$ if and only if for any map $G: D \rightarrow E$ satisfying

(1) $G$ has closed [resp., open] values, and

(2) $G$ is a KKM map,

$\{G(y)\}_{y \in D}$ has the finite intersection property. Further, if

(3) $\bigcap_{z \in M} \overline{G(z)}$ is compact for some $M \in\langle D\rangle$, then we have

$$
\bigcap_{y \in D} \overline{G(y)} \neq \emptyset .
$$

Proof. The first part is a simple consequence of Theorem 1 or the definition. Now the whole intersection property follows from the compactness.

Remarks. 1. You may prefer to adopt "compactly" closed [resp., open] values in (1). This is impractical and superfluous. In fact, replacing the topology of $E$ by its compactly generated extension, we can eliminate that kind of inadequate terminology; see [37].

2. Some authors call $G$ a transfer closed map when $\bigcap_{y \in D} \overline{G(y)}=\bigcap_{y \in D} G(y)$. In this case, the conclusion of Corollary 1.2 becomes $\bigcap_{y \in D} G(y) \neq \emptyset$.

From Theorem 1, we have the following prototype of the Alexandroff-Pasynkoff theorem [3]:

For $D:=\left\{a_{0}, a_{1}, \ldots, a_{n}\right\}$, we denote as follows:

$$
D_{0}:=\left\{a_{0}, \ldots, a_{n-1}\right\}, D_{i}:=\left\{a_{0}, \ldots, \widehat{a_{i-1}}, \ldots, a_{n}\right\}
$$

for $1 \leq i \leq n$. 
We say that $\Gamma:\langle D\rangle \multimap E$ is monotone if for any $A, B \in\langle D\rangle, A \subset B$ implies $\Gamma_{A} \subset \Gamma_{B}$. This holds automatically for a classical convexity spaces.

Corollary 1.2. Let $(E, D ; \Gamma)$ be an abstract convex topological space such that $\Gamma$ is monotone and $D$ is as above, $F \in \mathfrak{K C}(E, E)$ [resp., $F \in \mathfrak{K O}(E, E)]$, and $T: D \multimap E$ a map with nonempty closed [resp., open] values such that

(1) $F(E)=T(D)$ and

(2) $F\left(\Gamma_{D_{i}}\right) \subset T\left(a_{i}\right)$ for $0 \leq i \leq n$.

Then $\bigcap_{i=0}^{n} T\left(a_{i}\right) \neq \emptyset$.

Proof. We show that $T$ is a KKM map with respect to $F$. Let $N \in\langle D\rangle$. If $N=D$, then $F\left(\Gamma_{N}\right) \subset F(E)=T(N)$ by (1). Suppose that $N \subsetneq D$. Then, by (2) and monotonicity,

$$
F\left(\Gamma_{N}\right) \subset F\left(\Gamma_{D_{j}}\right) \subset T\left(a_{j}\right) \text { for some } a_{j} \in N,
$$

and hence

$$
F\left(\Gamma_{N}\right) \subset \bigcup\left\{T\left(a_{i}\right) \mid a_{i} \in N\right\}=T(N) .
$$

Now the conclusion follows from Theorem 1 .

Remark. For $E=D=\Delta_{n}, \Gamma=c o$, and $F=1_{E} \in \mathfrak{K C}(E, E) \cap \mathfrak{K O}(E, E)$, Corollary 1.2 reduces to the Alexandroff-Pasynkoff theorem [3], which was applied to the essentiality of the identity map of a simplex.

From Theorem 1, we have another finite intersection property as follows:

Theorem 2. Let $(E, D ; \Gamma)$ be an abstract convex space, $Z$ a set, and $F \in$ $\mathfrak{K}(E, Z)$. Let $G: D \multimap Z$ and $H: E \multimap Z$ be maps satisfying

(2.1) for each $x \in E, F(x) \subset H(x)$; and

(2.2) for each $z \in F(E), M \in\left\langle D \backslash G^{-}(z)\right\rangle$ implies $\Gamma_{M} \subset E \backslash H^{-}(z)$.

Then $F(E) \cap \bigcap\{G(y) \mid y \in N\} \neq \emptyset$ for each $N \in\langle D\rangle$.

Proof. It suffices to show that (2.1) and (2.2) imply (1.1). Suppose that there exists an $N \in\langle D\rangle$ such that $F\left(\Gamma_{N}\right) \notin \subset G(N)$; that is, there exist $x \in \Gamma_{N}$ and $z \in F(x)$ such that $z \notin G(y)$ for all $y \in N$. In other words, $N \in\left\langle D \backslash G^{-}(z)\right\rangle$. By (2.2), $\Gamma_{N} \subset X \backslash H^{-}(z)$. Since $x \in \Gamma_{N}$, we have $x \notin H^{-}(z)$ or $z \notin H(x)$. Since $z \in F(x)$, this contradicts (2.1). This completes our proof.

Remark. As in Theorem 1 , if $Z$ is a topological space and $G$ is open-valued [resp., closed-valued], then we can assume $F \in \mathfrak{K O}(E, Z)[$ resp., $F \in \mathfrak{K C}(E, Z)]$.

\section{Coincidence and matching theorems}

From Theorem 2, as in [41], we have the following coincidence theorem:

Theorem 3. Let $(E, D ; \Gamma)$ be an abstract convex space, $Z$ a set, $S: D$-o $Z, T: E \rightarrow Z$ maps, and $F \in \mathfrak{K}(E, Z)$. Suppose that

(3.1) for each $z \in F(E), \operatorname{co}_{\Gamma} S^{-}(z) \subset T^{-}(z)$ [that is, $T^{-}(z)$ is $\Gamma$-convex relative to $\left.S^{-}(z)\right]$; and

(3.2) $F(E) \subset S(N)$ for some $N \in\langle D\rangle$. 
Then there exists an $\bar{x} \in E$ such that $F(\bar{x}) \cap T(\bar{x}) \neq \emptyset$.

Proof of Theorem 3 using Theorem 2. Suppose that $F(x) \cap T(x)=\emptyset$ for all $x \in E$. Let $G(y)=Z \backslash S(y)$ for $y \in D$ and $H(x)=Z \backslash T(x)$ for $x \in E$. Then all of the requirements of Theorem 2 are satisfied. Therefore, there exists a $z_{N} \in F(E) \cap \bigcap\{G(y) \mid y \in N\}$ for each $N \in\langle D\rangle$; that is, for each $N \in\langle D\rangle$, $z_{N} \in F(E)$ but $z_{N} \notin S(N)$. This contradicts (3.2).

We can also give a proof from the definition of $\mathfrak{K}$ :

Proof. For each $y \in D$, define $R(y):=F(E) \backslash S(y)$. Then $\bigcap_{y \in N} R(y)=$ $F(E) \backslash \bigcup_{y \in N} S(y)=\emptyset$ by (3.2), that is, the values of the map $R: D \multimap Z$ does not have the finite intersection property. Since $F \in \mathfrak{K}(E, Z), F\left(\Gamma_{M}\right) \not \subset R(M)$ for some $M \in\langle D\rangle$. Hence, there exist $\bar{x} \in \Gamma_{M}$ and $\bar{z} \in F(\bar{x})$ such that $\bar{z} \notin R(M)$. Then, $\bar{z} \in S(y)$ and hence $y \in S^{-}(\bar{z})$ for all $y \in M$. This implies $\bar{x} \in \Gamma_{M} \subset T^{-}(\bar{z})$ by (3.1). Therefore, $\bar{z} \in F(\bar{x}) \cap T(\bar{x})$.

Remark. If $Z$ is a topological space and $S$ has open [resp., closed] values, then $R$ has relatively closed [resp., open] values in $F(E)$. Then we can assume $F \in \mathfrak{K C}(E, Z)$ [resp., $F \in \mathfrak{K O}(E, Z)]$ in Theorem 3 .

From Theorem 3, we obtain the following Ky Fan type matching theorem:

Theorem 4. Let $(E, D ; \Gamma)$ be an abstract convex space, $Z$ a set, $S: D \multimap Z$, and $F \in \mathfrak{K}(E, Z)$ satisfying (3.2). Then there exists an $M \in\langle D\rangle$ such that $F\left(\Gamma_{M}\right) \cap \cap\{S(y) \mid y \in M\} \neq \emptyset$.

Proof. Let $H: Z \multimap E$ and $T: E \multimap Z$ be defined by $H(z):=\operatorname{cor}_{\Gamma} S^{-}(z)$ for $z \in Z$ and $T(x):=H^{-}(x)$ for $x \in E$. Then (3.1) is satisfied, hence $T$ and $F$ have a coincidence point $x_{0} \in E$; that is, $T\left(x_{0}\right) \cap F\left(x_{0}\right) \neq \emptyset$. For $z \in T\left(x_{0}\right) \cap F\left(x_{0}\right)$, we have $x_{0} \in T^{-}(z)=\operatorname{co}_{\Gamma} S^{-}(z)=\bigcup\left\{\Gamma_{M} \mid M \in\left\langle S^{-}(z)\right\rangle\right\}$, and hence $x_{0} \in \Gamma_{M}$ for some $M \in\left\langle S^{-}(z)\right\rangle$. Since $z \in S(y)$ for all $y \in M$, we have $z \in F\left(x_{0}\right) \cap \cap\{S(y) \mid y \in M\}$. This completes our proof.

Remark. The origin of Theorem 4 goes back to Ky Fan $[16,17]$ for a convex space $X=D$. For applications, see also [28, 29].

Theorem 4 can be stated in its contrapositive form and in terms of the complement $G(x)$ of $S(x)$ in $Z$. Then we obtain Theorem 1 .

Proof of Theorem 1 using Theorem 4. Suppose the conclusion does not hold. Then for some $N \in\langle D\rangle, F(E) \subset S(N)$ where $S(y):=Z \backslash G(y)$ for $y \in$ $D$. Therefore, by Theorem 4, there exists an $M \in\langle D\rangle$ such that $F\left(\Gamma_{M}\right) \cap$ $\bigcap\{S(y) \mid y \in M\} \neq \emptyset$; that is, $F\left(\Gamma_{M}\right) \not \subset G(M)$. This contradicts (1.1).

Therefore Theorems 1-4 are equivalent. 


\section{Analytic alternatives}

There are many equivalent and useful formulations of Theorems 1-4 in the KKM theory. In this section, we give some analytic alternatives.

We begin, in this section, with the following useful reformulation of Theorem 3:

Theorem 5. Let $(E, D ; \Gamma)$ be an abstract convex space, $Z$ a set, $F \in \mathfrak{K}(E, Z)$, $A, B \subset C$ sets and $f: E \times Z \rightarrow C, g: D \times Z \rightarrow C$ functions. Suppose that

(5.1) for each $z \in F(E)$ and $M \in\langle\{y \in D \mid g(y, z) \in A\}\rangle$, we have

$$
\Gamma_{M} \subset\{x \in E \mid f(x, z) \in B\} \text {. }
$$

Then either

(a) for each $N \in\langle D\rangle$, there exists a $z_{N} \in F(E)$ such that $g\left(y, z_{N}\right) \notin A$ for all $y \in N$; or

(b) there exists an $(\hat{x}, \hat{z}) \in F$ such that $f(\hat{x}, \hat{z}) \in B$.

Proof of Theorem 5 using Theorem 3. Consider the maps $S: D \multimap Z$ and $T: E \multimap Z$ given by

$$
S(y):=\{z \in Z \mid g(y, z) \in A\} \quad \text { for } y \in D
$$

and

$$
T(x):=\{z \in Z \mid f(x, z) \in B\} \quad \text { for } x \in E .
$$

Then (5.1) implies (3.1) in Theorem 3. Suppose that (a) does not hold. Then, for some $N \in\langle D\rangle$ and for each $z \in F(E)$ there exists an $y \in N$ such that $g(y, z) \in A$; that is, $F(E) \subset S(N)$. Hence (3.2) in Theorem 3 holds. Therefore, by Theorem $3, F$ and $T$ have a coincidence point $\hat{x} \in X$ with $F(\hat{x}) \cap T(\hat{x}) \neq \emptyset$; that is, (b) holds.

Remarks. 1. If $Z$ is a topological space and $S$ has open [resp., closed] values, then we can assume $F \in \mathfrak{K C}(E, Z)$ [resp., $F \in \mathfrak{K O}(E, Z)]$ as in Theorem 3 .

2. The first form of Theorem 5 is due to Lassonde [26]. For another form, see $[31,47]$.

From Theorem 5, we have the following analytic alternative, which is a basis of various equilibrium problems:

Theorem 6. Let $(E, D ; \Gamma)$ be an abstract convex space, $Z$ a set, $F \in \mathfrak{K}(E, Z)$, $\alpha, \beta \in \mathbb{R}$, and $f: E \times Z \rightarrow \overline{\mathbb{R}}, g: D \times Z \rightarrow \overline{\mathbb{R}}$ extended real-valued functions. Suppose that

(6.1) for each $z \in F(E)$ and $M \in\langle\{y \in D \mid g(y, z)>\alpha\}\rangle$, we have

$$
\Gamma_{M} \subset\{x \in E \mid f(x, z)>\beta\} \text {. }
$$

Then either

(a) for each $N \in\langle D\rangle$, there exists a $z_{N} \in F(E)$ such that $g\left(y, z_{N}\right) \leq \alpha$ for all $y \in N$; or

(b) there exists an $(\hat{x}, \hat{z}) \in F$ such that $f(\hat{x}, \hat{z})>\beta$. 
Proof. Put $C:=\overline{\mathbb{R}}, A:=(\alpha, \infty]$, and $B:=(\beta, \infty]$ in Theorem 5 .

Remarks. 1. If $Z$ is a topological space and $\{z \in Z \mid g(y, z)>\alpha\}$ is open [resp., closed] for each $y \in D$, then we can assume $F \in \mathfrak{K C}(E, Z)$ [resp., $F \in \mathfrak{K O}(E, Z)$ ] as in Theorem 5 .

2. If $E=D=Z$ is a compact convex space, Theorem 6 reduces to the principal result of Ben-El-Mechaiekh et al. [5, 6], where this result is applied to variational inequalities of Hartman-Stampacchia and of Browder and a generalization of the Ky Fan minimax inequality [15].

\section{Minimax inequalities}

From Theorem 6, we clearly have the following generalized form of the $\mathrm{Ky}$ Fan minimax inequality [15]:

Theorem 7. Under the hypothesis of Theorem 6, if

$$
\alpha=\beta=\sup \{f(x, z) \mid(x, z) \in F\},
$$

then for each $N \in\langle D\rangle$,

(c) there exists a $z_{N} \in F(E)$ such that

$$
g\left(y, z_{N}\right) \leq \sup _{(x, z) \in F} f(x, z) \quad \text { for all } y \in N ; \text { and }
$$

(d) we have the following minimax inequality

$$
\inf _{z \in F(E)} \sup _{y \in N} g(y, z) \leq \sup _{(x, z) \in F} f(x, z) .
$$

In order to show that Theorem 7 is equivalent to any of Theorems $1-6$, we give the following:

Proof of Theorem 2 using Theorem 7. Define real functions $g: D \times Z \rightarrow \mathbb{R}$ and $f: X \times Z \rightarrow \mathbb{R}$ by

$$
g(y, z):= \begin{cases}0 & \text { if } z \in G(y) \\ 1 & \text { otherwise }\end{cases}
$$

for $(y, z) \in D \times Z$ and

$$
f(x, z)= \begin{cases}0 & \text { if } z \in H(x) \\ 1 & \text { otherwise }\end{cases}
$$

for $(x, z) \in E \times Z$. Put $\alpha=\beta=0$. Then (2.1) implies (6.1). In fact, $M \in\langle\{y \in D \mid g(y, z)>0\}\rangle=\langle\{y \in D \mid g(y, z)=1\}\rangle=\left\langle D \backslash G^{-}(z)\right\rangle$ implies $\Gamma_{M} \subset E \backslash H^{-}(z)=\{x \in E \mid f(x, z)=1\}=\{x \in E \mid f(x, z)>0\}$. Since $\sup \{f(x, z) \mid(x, z) \in F\} \leq \sup \{f(x, z) \mid(x, z) \in H\}=0$ by (2.1) and the definition of $f, \sup \{f(x, z) \mid(x, z) \in F\}=0$. Therefore, by Theorem 7 , for each $N \in\langle D\rangle$, there exists a $z_{N} \in F(X)$ such that

$$
g\left(y, z_{N}\right) \leq \sup _{(x, z) \in F} f(x, z)=0 \text { for all } y \in N .
$$


Hence $g\left(y, z_{N}\right)=0$ for all $y \in N$; that is, $z_{N} \in G(y)$ for all $y \in N$. Therefore,

$$
F(E) \cap \bigcap\{G(y) \mid y \in N\} \neq \emptyset \text {. }
$$

This completes our proof.

Remarks. 1. Conclusion (d) can be written as follows: For each $N \in\langle D\rangle$,

$$
\inf _{z \in F(E)} \sup _{y \in N} g(y, z) \leq \inf _{F \in \mathcal{K}(E, Z)} \sup _{(x, z) \in F} f(x, z)
$$

2. If $Z$ is a topological space and $\{z \in Z \mid g(y, z)>\alpha\}$ is open [resp., closed] for each $y \in D$, then we can assume $F \in \mathfrak{K C}(E, Z)[$ resp., $F \in \mathfrak{K O}(E, Z)]$ as in Theorem 6.

The KKM type theorem or some whole intersection theorem can be reformulated to minimax inequalities. The following minimax inequality is equivalent to Theorem 1:

Theorem 8. Let $(E, D ; \Gamma)$ be an abstract convex space, $Z$ a set, and $F \in$ $\mathfrak{K}(E, Z)$. Let $\phi: D \times Z \rightarrow \overline{\mathbb{R}}$ be an extended real function and $\gamma \in \overline{\mathbb{R}}$ such that

(8.1) for each $N \in\langle D\rangle$ and $z \in F\left(\Gamma_{N}\right), \min \{\phi(y, z) \mid y \in N\} \leq \gamma$.

Then for each $N \in\langle D\rangle$,

(a) there exists a $z_{N} \in F(E)$ such that

$$
\phi\left(y, z_{N}\right) \leq \gamma \quad \text { for all } y \in N ; \text { and }
$$

(b) if $E=D$ and $\gamma=\sup \{\phi(x, z) \mid(x, z) \in F\}$, then we have the minimax inequality:

$$
\inf _{z \in F(E)} \sup _{y \in N} \phi(y, z) \leq \sup _{(x, z) \in F} \phi(x, z) .
$$

Proof of Theorem 8 using Theorem 1. Let $G(y):=\{z \in Z \mid \phi(y, z) \leq \gamma\}$ for $y \in D$. We show that (8.1) implies (1.1). Suppose that there exists an $N \in\langle D\rangle$ such that $F\left(\Gamma_{N}\right) \not \subset G(N)$. Choose a $z \in F\left(\Gamma_{N}\right)$ such that $z \notin G(N)$, whence $\phi(y, z)>\gamma$ for all $y \in N$. Then $\min _{y \in N} \phi(y, z)>\gamma$, which contradicts (8.1). Therefore, by Theorem 1, for each $N \in\langle D\rangle$, there exists a $z_{N} \in F(E)$ such that $z_{N} \in G(y)$ for all $y \in N$; that is, $\phi\left(y, z_{N}\right) \leq \gamma$ for all $y \in N$. This completes the proof of (a). Note that (b) clearly follows from (a).

Proof of Theorem 1 using Theorem 8. Define $\phi: D \times Z \rightarrow \overline{\mathbb{R}}$ by

$$
\phi(y, z):= \begin{cases}0 & \text { if } z \in G(y) \\ 1 & \text { otherwise }\end{cases}
$$

for $(y, z) \in D \times Z$. Put $\gamma=0$ in Theorem 8. We show that (1.1) implies (8.1). In fact, suppose that there exist an $N \in\langle D\rangle$ and a $z \in F\left(\Gamma_{N}\right)$ such that $\min \{\phi(y, z) \mid y \in N\}>0$. Then $z \notin G(y)$ for all $y \in N$; that is, $F\left(\Gamma_{N}\right) \not \subset G(N)$, which contradicts (1.1). Therefore, all of the requirements of Theorem 8 are satisfied. Hence, for each $N \in\langle D\rangle$, there exists a $z_{N} \in F(E)$ such that $\phi\left(y, z_{N}\right)=0$ for all $y \in N$; that is, $z_{N} \in \bigcap\{G(y) \mid y \in N\}$. This completes our proof. 
Remarks. 1. In the proof of the equivalency of Theorems 1 and 8 we used that (8.1) is equivalent to

$(8.1)^{\prime}$ the map $y \mapsto G(y)=\{z \in Z \mid \phi(y, z) \leq \gamma\}$ satisfies Condition (1.1).

2. As in Theorem 1 , if $Z$ has any topology and $G(y):=\{z \in Z \mid \phi(y, z) \leq \gamma\}$ is open [resp., closed] for each $y \in D$, then we can assume $F \in \mathfrak{K O}(E, Z)$ [resp., $F \in \mathfrak{K C}(E, Z)]$.

\section{Geometric or section properties}

In 1961, Fan [11] gave a "geometric" lemma which is the geometric equivalence of his version of the KKM theorem. In many of his works in the KKM theory, Fan actually based his arguments mainly on the geometric or section property of a convex space. We now deduce two geometric forms of Theorem 2. The first one is as follows:

Theorem 9. Let $(E, D ; \Gamma)$ be an abstract convex space, $Z$ a set, $F \in \mathfrak{K}(E, Z)$, $A \subset B \subset E \times Z$, and $C \subset D \times Z$. Suppose that

(9.1) for each $z \in F(X)$ and $M \in\langle\{y \in D \mid(y, z) \notin C\}\rangle$, we have

$$
\Gamma_{M} \subset\{x \in X \mid(x, z) \notin B\} ;
$$

(9.2) A contains the graph of $F$.

Then for each $N \in\langle D\rangle$, there exists a $z_{N} \in F(X)$ such that $N \times\left\{z_{N}\right\} \subset C$.

Proof of Theorem 9 using Theorem 2. For each $y \in D$, let

$$
G(y):=\{z \in Z \mid(y, z) \in C\},
$$

and, for each $x \in X$, let

$$
H(x):=\{z \in Z \mid(x, z) \in B\} .
$$

Since $A \subset B,(9.1)$ and (9.2) imply (2.1) and (2.2). Hence, $G$ satisfies all of the requirements of Theorem 2 . Therefore, for each $N \in\langle D\rangle$, we have

$$
F(E) \cap \bigcap\{G(y) \mid y \in N\} \neq \emptyset .
$$

Hence, for each $N \in\langle D\rangle$, there exists a $z_{N} \in F(E)$ such that $z_{N} \in \bigcap\{G(y) \mid y \in$ $N\}$; that is, $N \times\left\{z_{N}\right\} \subset C$.

Remarks. 1. As in Theorem 2, if $Z$ has any topology and $G(y):=\{z \in$ $Z \mid(y, z) \in C\}$ is open [resp., closed] for each $y \in D$, then we can assume $F \in \mathfrak{K O}(E, Z)$ [resp., $F \in \mathfrak{K C}(E, Z)]$.

2. If $E=D$ is a convex subset of a topological vector space and if $A=B=$ $C$, Theorem 9 reduces to Fan's 1961 Lemma [11, Lemma 4]. He obtained his result from his own generalization of the KKM principle and applied it to a direct proof of the Tychonoff fixed point theorem. Other interesting applications of his useful lemma to fixed points, minimax theorems, equilibrium points, extension of monotone sets, potential theory, etc. have been made by Fan [13] and many others; see [31, 35]. 
The following form of Theorem 9 is also widely used in the KKM theory:

Theorem 10. Let $(E, D ; \Gamma)$ be an abstract convex space, $Z$ a set, $F \in \mathfrak{K}(E, Z)$, $B \subset A \subset E \times Z$, and $C \subset D \times Z$. Suppose that

(10.1) for each $z \in F(E)$ and $M \in\langle\{y \in D \mid(y, z) \in C\}\rangle$, we have $\Gamma_{M} \subset$ $\{x \in E \mid(x, z) \in B\} ;$ and

(10.2) for some $N \in\langle D\rangle$, there exists an $y \in N$ such that $(y, z) \in C$ for all $z \in F(E)$.

Then there exist an $x_{0} \in E$ and $a z_{0} \in F\left(x_{0}\right)$ such that $\left(x_{0}, z_{0}\right) \in A$.

Proof of Theorem 10 using Theorem 9. Consider Theorem 9 with the complements $\left(A^{c}, B^{c}, C^{c}\right)$ instead of $(A, B, C)$. Then $(9.1)$ is satisfied automatically. Since (10.2) is the negation of the conclusion of Theorem 9 , we should have the negation of (9.2). Therefore, the conclusion follows.

Proof of Theorem 3 using Theorem 10. Let $A=B$ be the graph of $T$ and $C$ the graph of $S$. Then (3.1) and (3.2) in Theorem 3 imply (10.1) and (10.2). Therefore, by Theorem 10 , there exists an $\left(x_{0}, z_{0}\right) \in F$ such that $\left(x_{0}, z_{0}\right) \in A$; that is, $F$ and $T$ have a coincidence point.

Remark. If $E=D$ is a convex subset of a topological vector space and if $A=B=C$, Theorem 10 reduces to Fan [15, Theorem 2].

Consequently, Theorems 1-10 are all equivalent to each other. Note that each of Theorems 1-10 is a kind of metatheorems which can be turned into a concrete theorem for a special type of abstract convex spaces and a particular map $F \in \mathfrak{K C}(E, Z)$ or $F \in \mathfrak{K O}(E, Z)$. For example, for a $G$-convex space $(X, D ; \Gamma)$, a Hausdorff space $Z$, and a map $F \in \mathfrak{A}_{c}^{\kappa}(X, Z)$, Theorems $1-10$ imply the main results of the generalized convex space theory in [47].

\section{The Fan-Browder type fixed point theorems}

From Theorem 3, as in [41], we have the following prototype of the FanBrowder fixed point theorem:

Theorem 11. Let $(E, D ; \Gamma)$ be an abstract convex topological space, and $G$ : $E \multimap D, F: E \multimap E$ maps satisfying

(11.1) for each $x \in E$, $\operatorname{cor}_{\Gamma} G(x) \subset F(x)$;

(11.2) $E=G^{-}(N)$ for some $N \in\langle D\rangle$; and

(11.3) $G^{-}$has open [resp., closed] values.

If the identity map $1_{E} \in \mathfrak{K C}(E, E)\left[\right.$ resp., $\left.1_{E} \in \mathfrak{K O}(E, E)\right]$, then $F$ has a fixed point $\bar{x} \in E$, that is, $\bar{x} \in F(\bar{x})$.

Proof. In Theorem 3 , let $S:=G^{-}, T:=F^{-}$, and $F:=1_{E}$.

Any binary relation $R$ in a set $X$ can be regarded as a map $T: X \multimap X$ and conversely by the following obvious way:

$$
y \in T(x) \text { if and only if }(x, y) \in R .
$$


Therefore, a point $x_{0} \in X$ is called a maximal element of a map $T$ if $T\left(x_{0}\right)=\emptyset$.

The Fan-Browder type fixed point theorem is used by Borglin and Keiding [7] and Yannelis and Prabhakar [51] to the existence of maximal elements in mathematical economics.

From Theorem 11, we have the following maximal element theorem:

Corollary 11.1. Let $(E, D ; \Gamma)$ be a abstract convex topological space and $G$ : $E \multimap D, F: E \multimap E$ maps satisfying

(1) for each $x \in E, \operatorname{co}_{\Gamma} G(x) \subset F(x)$;

(2) $F^{-}(E) \subset G^{-}(N)$ for some $N \in\langle D\rangle$;

(3) $G^{-}$has open $[$resp., closed] values; and

(4) $x \notin F(x)$ for all $x \in E$.

If the identity map $1_{E} \in \mathfrak{K C}(E, E)$ [resp., $\left.1_{E} \in \mathfrak{K O}(E, E)\right]$, then $F$ has a maximal element $\bar{x} \in E$, that is, $F(\bar{x})=\emptyset$.

Proof. Note that (1) and (3) are same to conditions (11.1) and (11.3), resp. Suppose that $F(x) \neq \emptyset$ for each $x \in E$. Then $E=F^{-}(E)=\bigcup\left\{F^{-}(y) \mid y \in E\right\}$. By (2), condition (11.2) holds. Therefore, by Theorem 11, $F$ has a fixed point. This violates (4).

For a $G$-convex space, Corollary 11.1 generalizes results in [37]. Note that the conclusions of Theorem 11 and Corollary 11.1 follow from the KKM theorem $1_{E} \in \mathfrak{K} \mathfrak{C}(E, E)$ [resp., $\left.1_{E} \in \mathfrak{K O}(E, E)\right]$.

Corollary 11.2. Let $(X, D ; \Gamma)$ be a G-convex space, and $S: X \multimap D, T$ : $X \multimap X$ maps satisfying

(1) for each $x \in X, \operatorname{co}_{\Gamma} S(x) \subset T(x)$;

(2) $E=S^{-}(N)$ for some $N \in\langle D\rangle$; and

(3) $S^{-}(y)$ is open [resp., closed] for each $y \in D$.

Then $H$ has a fixed point $\bar{x} \in E$.

Proof. In Theorem 11, let $G:=S$ and $F:=T$. Since the identity map $1_{X} \in$ $\mathfrak{K C}(X, X) \cap \mathfrak{K O}(X, X)$, the conclusion follows from Theorem 11 .

Remarks. 1. For a compact convex subset $X=D$ of a topological vector space $E$, if $S=T$ and $T^{-}(y)$ itself is open, then Corollary 11.2 reduces to the Browder fixed point theorem [8].

2. Note that the Browder theorem is a reformulation of Fan's geometric lemma [11] in the form of a fixed point theorem and its proof was based on the Brouwer fixed point theorem and the partition of unity argument. Therefore it is also called the Fan-Browder fixed point theorem.

3. Browder [8] applied his theorem to a systematic treatment of the interconnections between multi-valued fixed point theorems, minimax theorems, variational inequalities, and monotone extension theorems.

4. For further developments on generalizations and applications of the FanBrowder theorem, we refer to Park [35, 38].

Motivated by Horvath [21, 22], we define the following: 
Definition. For a given abstract convex space $(E, D ; \Gamma)$ and a topological space $X$, a map $H: X \multimap E$ is called a $\Phi$-map (or a Fan-Browder map) if there exists a map $G: X \multimap D$ such that

(i) for each $x \in X, \operatorname{co}_{\Gamma} G(x) \subset H(x)$; and

(ii) $X=\bigcup\left\{\operatorname{Int} G^{-\cdots}(y) \mid y \in D\right\}$.

Definitions. An abstract convex uniform space $(E, D ; \Gamma ; \mathcal{U})$ is an abstract convex space with a basis $\mathcal{U}$ of a uniform structure of $E$.

In $(E, D ; \Gamma ; \mathcal{U})$, a subset $Z$ of $E$ is called a $\Phi$-set if, for any entourage $U \in \mathcal{U}$, there exists a $\Phi$-map $H: Z \rightarrow E$ such that $\operatorname{Gr}(H) \subset U$. If $E$ itself is a $\Phi$-set, then it is called a $\Phi$-space.

A point $x \in E$ is called a $U$-fixed point of a map $F: E \multimap E$ if $F(x) \cap U[x] \neq \emptyset$. The map $F$ is said to have the almost fixed point property whenever it has a $U$-fixed point for any $U \in \mathcal{U}$.

Examples. We give the following examples of $\Phi$-sets; see [42].

1. Any locally convex subset of a t.v.s. $E$ is a $\Phi$ set in $E$ (for example, every nonempty subset of a locally convex t.v.s. and every nonempty subset of a locally convex set).

2. Horvath [21] gave examples of $\Phi$-spaces in the class of his $C$-spaces as follows:

(1) A particular type of uniform spaces including locally convex t.v.s.

(2) Convex metric spaces in the sense of Takahashi with a metric satisfying certain property.

3. Any subset of the Zima type in a $G$-convex uniform space $(X \supset D ; \Gamma ; \mathcal{U})$ such that every singleton is $\Gamma$-convex is a $\Phi$-set.

4. For a locally $G$-convex space $(X \supset D ; \Gamma ; \mathcal{U})$, any nonempty subset $Y$ of $X$ is a $\Phi$-set. A locally $G$-convex space $(X \supset D ; \Gamma ; \mathcal{U})$ is a $\Phi$-space.

5. A metric $G$-convex space $(X \supset D ; \Gamma$ ) is a $\Phi$-space whenever (1) $D$ is dense in $X$ and (2) every open ball is $\Gamma$-convex.

Now we have the following almost fixed point theorem:

Theorem 12. Let $(E, D ; \Gamma ; \mathcal{U})$ be an abstract convex uniform space, and $F \in$ $\mathfrak{K C}(E, E)$ be a compact map. If $\overline{F(E)}$ is a $\Phi$-set, then $F$ has the almost fixed point property.

Proof. Since $Z:=\overline{F(E)}$ is a $\Phi$-set, for any entourage $U \in \mathcal{U}$, there exists a $\Phi$ map $H: Z \multimap E$ such that $\operatorname{Gr}(H) \subset U$, that is, there exists a map $G: Z \multimap D$ such that

(i) for each $x \in Z, \operatorname{co}_{\Gamma} G(x) \subset H(x)$; and

(ii) $Z=\bigcup\left\{\operatorname{Int} G^{-}(y) \mid y \in D\right\}$.

Since $Z$ is compact, $F(E) \subset Z=\bigcup\left\{\operatorname{Int} G^{-}(y) \mid y \in N\right\}$ for some $N \in\langle D\rangle$. Let $S:=G^{-}$and $T:=H^{-}$. Since $F \in \mathfrak{K C}(E, Z)$, by Theorem 3 , there exists 
an $\bar{x} \in E$ such that $F(\bar{x}) \cap H^{-}(\bar{x}) \neq \emptyset$. Since $\operatorname{Gr}(H) \subset U$ and $H^{-}(\bar{x})=\{z \in$ $Z \mid \bar{x} \in H(z)\}$, we have $H^{-}(\bar{x}) \subset U^{-}[\bar{x}]$. Since we may assume $U$ is symmetric, $F(\bar{x}) \cap U[\bar{x}] \neq \emptyset$. This completes our proof.

Corollary 12.1. Under the hypothesis of Theorem 12, further if $(E, \mathcal{U})$ is separated and if $F$ is closed, then it has a fixed point.

Lemma 3. Let $(E, D ; \Gamma)$ be an abstract convex space, $Z, W$ two sets, $F \in$ $\mathfrak{K}(E, Z)$ and $f: Z \rightarrow W$ a function. Then $f F \in \mathfrak{K}(E, W)$.

Proof. Let $G: D \multimap W$ be a map satisfying $f F\left(\Gamma_{A}\right) \subset G(A)$ for all $A \in\langle D\rangle$. Then $F\left(\Gamma_{A}\right) \subset f^{-1} G(A)$ for all $A \in\langle D\rangle$. Since $F \in \mathfrak{K}(E, Z)$, the family $\left\{f^{-1} G(y)\right\}_{y \in D}$ has the finite intersection property. Then, so does the family $\{G(y)\}_{y \in D}$. This shows $f F \in \mathfrak{K}(E, W)$.

Similarly we have the following:

Lemma 4. Let $(E, D ; \Gamma)$ be an abstract convex topological space and $Z$ a topological space. If $1_{E} \in \mathfrak{K C}(E, E)$, then $f \in \mathfrak{K C}(E, Z)$ for any continuous function $f: E \rightarrow Z$. This also holds for $\mathfrak{K O}$ instead of $\mathfrak{K C}$.

Similarly, we have the following:

Lemma 5. Let $(E, D ; \Gamma)$ be an abstract convex topological space, $Z, W$ two topological spaces, $F \in \mathfrak{K C}(E, Z)$ and $f: Z \rightarrow W$ a continuous function. Then $f F \in \mathfrak{K C}(E, W)$. This also holds for $\mathfrak{K O}$ instead of $\mathfrak{K C}$.

From Corollary 12.1 and Lemma 5, we have the following generalization of the Schauder-Tychonoff-Hukuhara fixed point theorem:

Corollary 12.2. Let $(E, D ; \Gamma ; \mathcal{U})$ be an abstract convex separated uniform space such that the identity map $1_{E} \in \mathfrak{K C}(E, E)$. If $f: E \rightarrow E$ is a continuous function such that $\overline{f(E)}$ is a compact $\Phi$-set in $E$, then $f$ has a fixed point.

Remark. Note that Amini et al. [4] obtained Theorem 12 and Corollaries 12.1 and 12.2 for the S-KKM class for closed-valued maps on a classical convexity space.

\section{Fixed point theorems for the better admissible class}

Our aim in this section is to show that, in the class of compact closed maps defined on a particular type of $G$-convex spaces, two subclasses $\mathfrak{B}$ and $\mathfrak{K C}$ are identical.

We begin this section with a short review of some results in a recent work of the author [42].

Definition. Let $(X, D ; \Gamma)$ be a $G$-convex space and $Z$ a topological space. In 2000 , we defined the better admissible class $\mathfrak{B}$ of maps from $X$ into $Z$ as follows: 
$F \in \mathfrak{B}(X, Z) \Longleftrightarrow F: X \multimap Z$ is a map such that for any $N \in\langle D\rangle$ with the cardinality $|N|=n+1$ and any continuous function $p: F\left(\Gamma_{N}\right) \rightarrow \Delta_{n}$, the composition

$$
\Delta_{n} \stackrel{\phi_{N}}{\rightarrow} \longrightarrow \Gamma_{N} \stackrel{\left.F\right|_{\Gamma_{N}}}{\rightarrow} \rightarrow F\left(\Gamma_{N}\right) \stackrel{p}{\rightarrow} \longrightarrow \Delta_{n}
$$

has a fixed point. Note that $\Gamma_{N}$ can be replaced by the compact set $\phi_{N}\left(\Delta_{n}\right)$.

Examples. Subclasses of the admissible class $\mathfrak{A}_{c}^{\kappa}$ are classes of continuous functions $\mathbb{C}$, the Kakutani maps $\mathbb{K}$ (with convex values and codomains are convex spaces), the Aronszajn maps $\mathbb{M}$ (with $R_{\delta}$ values), the acyclic maps $\mathbb{V}$ (with acyclic values), the Powers maps $\mathbb{V}_{c}$ (finite compositions of acyclic maps), the O'Neill maps $\mathbb{N}$ (continuous with values of one or $m$ acyclic components, where $m$ is fixed), the u.s.c. approachable maps $\mathbb{A}$ (whose domains and codomains are uniform spaces), admissible maps of Górniewicz, $\sigma$-selectionable maps of Haddad and Lasry, permissible maps of Dzedzej, the class $\mathbb{K}_{c}^{\sigma}$ of Lassonde, the class $\mathbb{V}_{c}^{\sigma}$ of Park et al., the u.s.c. approximable maps of Ben-El-Mechaiekh and Idzik, and many others.

Note that for a $G$-convex space $(X, D ; \Gamma)$ and any space $Y$, an admissible class $\mathfrak{A}_{c}^{\kappa}(X, Y)$ is a subclass of $\mathfrak{B}(X, Y)$ with some possible exceptions such as Kakutani maps. There are maps in $\mathfrak{B}$ not belonging to $\mathfrak{A}_{c}^{\kappa}$, for example, the connectivity map due to Nash and Girolo; see [34].

The following is known:

Lemma 6. Let $(X, D ; \Gamma)$ be a $G$-convex space and $Z$ a topological space. Then

(1) $\mathbb{C}(X, Z) \subset \mathfrak{A}_{c}^{\kappa}(X, Z) \subset \mathfrak{B}(X, Z)$;

(2) $\mathbb{C}(X, Z) \subset \mathfrak{K C}(X, Z) \cap \mathfrak{K O}(X, Z)$; and

(3) [24] $\mathfrak{A}_{c}^{\kappa}(X, Z) \subset \mathfrak{K C}(X, Z) \cap \mathfrak{K D}(X, Z)$ if $Z$ is Hausdorff.

For more general purposes, we introduce a generalized version of the admissibility of subsets of topological vector spaces as follows:

Definition. A $G$-convex uniform space $(X, D ; \Gamma ; \mathcal{U})$ is a $G$-convex space with a basis $\mathcal{U}$ of a uniform structure of $X$. A subset $Y$ of $X$ is said to be admissible (in the sense of Klee) if, for each nonempty compact subset $K$ of $Y$ and for each entourage $U \in \mathcal{U}$, there exists a continuous function $h: K \rightarrow Y$ satisfying

(1) $(x, h(x)) \in U$ for all $x \in K$;

(2) $h(K) \subset \Gamma_{N}$ for some $N \in\langle D\rangle$; and

(3) there exists a continuous function $p: K \rightarrow \Delta_{n}$ such that $h=\phi_{N} p$, where $\phi_{N}: \Delta_{n} \rightarrow \Gamma_{N}$ and $|N|=n+1$.

Definition. Let $(X, D ; \Gamma ; \mathcal{U})$ be a $G$-convex uniform space. A subset $K$ of $X$ is said to be Klee approximable if, for each entourage $U \in \mathcal{U}$, there exists a continuous function $h: K \rightarrow X$ satisfying conditions (1)-(3) in the preceding definition. Especially, for a subset $Y$ of $X, K$ is said to be Klee approximable into $Y$ whenever the range $h(K) \subset \Gamma_{N} \subset Y$ for some $N \in\langle D\rangle$ in condition (2). 
The following is recently given in [42]:

Lemma 7. In the class of $G$-convex uniform spaces $(X, D ; \Gamma ; \mathcal{U})$, the following hold:

(1) Any LG-space is of the Zima-Hadżić type.

(2) Every $L G$-space is locally $G$-convex whenever every singleton is $\Gamma$-convex.

(3) Any nonempty subset of a locally $G$-convex space is a $\Phi$-set.

(4) Any Zima-Hadžić type subset of a $G$-convex uniform space such that every singleton is $\Gamma$-convex is a $\Phi$-set.

(5) Every $\Phi$-space is admissible. More generally, every nonempty compact $\Phi$-subset is Klee approximable.

The following is the main result of [42]:

Theorem 13. Let $(X, D ; \Gamma ; \mathcal{U})$ be a $G$-convex uniform space, $Y$ a subset of $X$, and $F \in \mathfrak{B}(Y, Y)$ a map such that $F(Y)$ is Klee approximable into $Y$. Then $F$ has the almost fixed point property.

Further if $(X ; \mathcal{U})$ is separated and $F$ is closed and compact, then $F$ has a fixed point $x_{0} \in Y$.

For the class $\mathfrak{B}$, we have the following coincidence theorem:

Theorem 14. Let $(X, D ; \Gamma)$ be a $G$-convex space, $Z$ a Hausdorff space, and $F \in \mathfrak{B}(X, Z)$ a compact map. Let $S: D \multimap Z$ and $T: X \multimap Z$ satisfy the following:

(14.1) for each $y \in D, S(y)$ is open in $Z$;

(14.2) for each $z \in F(X)$, $\operatorname{co}_{5} S^{-}(z) \subset T^{-}(z)$; and

(14.3) $\overline{F(X)} \subset S(D)$.

Then $T$ and $F$ have a coincidence point $x_{0} \in X$; that is, $T\left(x_{0}\right) \cap F\left(x_{0}\right) \neq \emptyset$.

Proof. Since $\overline{F(X)}$ is compact and covered by open sets $S(y)$ by (14.1) and (14.3), there exists an $N \in\langle D\rangle$ such that $\overline{F(X)} \subset S(N)$. Let $N:=\left\{y_{0}, y_{1}\right.$, $\left.\ldots, y_{n}\right\} \in\langle D\rangle$. Then there exists a continuous function $\phi_{N}: \Delta_{n} \rightarrow \Gamma_{N}$ such that $\phi_{N}\left(\Delta_{J}\right) \subset \Gamma_{J}$ for each $J \in\langle N\rangle$. Let $\left\{\lambda_{i}\right\}_{i=0}^{n}$ be the partition of unity subordinated to the cover $\left\{S\left(y_{i}\right)\right\}_{i=0}^{n}$ of $\overline{F(X)} \supset F \phi_{N}\left(\Delta_{n}\right)$.

Define $p: F \phi_{N}\left(\Delta_{n}\right) \rightarrow \Delta_{n}$ by

$$
p(z):=\sum_{i=0}^{n} \lambda_{i}(z) e_{i}=\sum_{i \in N_{z}} \lambda_{i}(z) e_{i} \text { for } z \in F \phi_{N}\left(\Delta_{n}\right)
$$

where $i \in N_{z} \Longleftrightarrow \lambda_{i}(z) \neq 0 \Longrightarrow z \in S\left(y_{i}\right) \Longleftrightarrow y_{i} \in S^{-}(z)$. By (14.2), we have $\phi_{N} p(z) \in \phi_{N}\left(\Delta_{N_{z}}\right) \subset \Gamma_{N_{z}} \subset T^{-}(z)$ for each $z \in F \phi_{N}\left(\Delta_{n}\right)$; that is, $z \in\left(T \phi_{N} p\right)(z)$.

Since $F \in \mathfrak{B}(X, Z), p F \phi_{N}$ has a fixed point $a_{0} \in \Delta_{n}$; that is, $a_{0} \in$ $\left(p F \phi_{N}\right)\left(a_{0}\right)$. Put $x_{0}:=\phi_{N}\left(a_{0}\right)$. Since $p^{-1}\left(a_{0}\right) \subset\left(F \phi_{N}\right)\left(a_{0}\right)=F\left(x_{0}\right)$, for any $z \in p^{-1}\left(a_{0}\right)$, we have $z \in F \phi_{N}\left(\Delta_{n}\right),\left(\phi_{N} p\right)(z)=\phi_{N}\left(a_{0}\right)=x_{0}$, and $z \in\left(T \phi_{N} p\right)(z)=T\left(x_{0}\right)$. Therefore, $p^{-1}\left(a_{0}\right) \subset T\left(x_{0}\right)$ and hence $p^{-1}\left(a_{0}\right) \subset$ $F\left(x_{0}\right) \cap T\left(x_{0}\right)$. This completes our proof. 
Remarks. 1 . In case $(X, D ; \Gamma)$ is a convex space, Theorem 14 is obtained in [33], where particular forms and applications are noted.

2. If $Z$ is a normal topological space and $\overline{F(X)} \subset S(N)$ for some $N \in\langle D\rangle$, the conclusion still holds.

As we deduced Theorem 1 from Theorem 3 via Theorem 4, we obtain the following from Theorem 14:

Theorem 15. Let $(X, D ; \Gamma)$ be a $G$-convex space, $Z$ a Hausdorff space, and $F \in \mathfrak{B}(X, Z)$ a compact map. Let $G: D \rightarrow Z$ be a map such that

(15.1) for each $y \in D, G(y)$ is closed in $Z$;

(15.2) for any $N \in\langle D\rangle, F\left(\Gamma_{N}\right) \subset G(N)$.

Then for each $N \in\langle D\rangle, \overline{F(X)} \cap \bigcap\{G(y) \mid y \in N\} \neq \emptyset$.

Remark. Theorems 14, 15 and their equivalent formulations were first given in [47] for $\mathfrak{A}_{c}^{\kappa}$ instead of $\mathfrak{B}$.

Consider the following condition for a $G$-convex space $(X \supset D ; \Gamma)$ :

(*) $\Gamma_{\{x\}}=\{x\}$ for each $x \in D$; and, for each $N \in\langle D\rangle$ with the cardinality $|N|=n+1$, there exists a continuous function $\phi_{N}: \Delta_{n} \rightarrow \Gamma_{N}$ such that $\phi_{N}\left(\Delta_{n}\right)=\Gamma_{N}$ and that $J \in\langle N\rangle$ implies $\phi_{N}\left(\Delta_{J}\right)=\Gamma_{J}$.

Note that every convex space satisfies condition $(*)$.

Now we are going to show the following:

Theorem 16. Let $(X, D ; \Gamma)$ be a $G$-convex space and $Z$ a topological space.

(1) If $Z$ is a Hausdorff space, then every compact map $F \in \mathfrak{B}(X, Z)$ belongs to $\mathfrak{K C}(X, Z)$.

(2) If $F: X \rightarrow Z$ is a closed map such that $F \phi_{N} \in \mathfrak{K C}\left(\Delta_{n}, Z\right)$ for any $N \in\langle D\rangle$ with the cardinality $|N|=n+1$, then $F \in \mathfrak{B}(X, Z)$.

(3) In the class of closed maps defined on a $G$-convex space $(X \supset D ; \Gamma)$ satisfying condition $(*)$ into a space $Z$, a map $F \in \mathfrak{K C}(X, Z)$ belongs to $\mathfrak{B}(X, Z)$.

Proof. (1) This is a restatement of Theorem 15.

(2) Let $p: F\left(\phi_{N}\left(\Delta_{n}\right)\right) \rightarrow \Delta_{n}$ be a continuous function. Since $F \phi_{N} \in$ $\mathfrak{K C}\left(\Delta_{n}, Z\right)$, by Lemma $3, p F \phi_{N} \in \mathfrak{K C}\left(\Delta_{n}, \Delta_{n}\right)$. Since $p F \phi_{N}$ is closed and $\Delta_{n}$ is a compact $\Phi$-space, by Corollary $12.1, p F \phi_{N}$ has a fixed point. Hence $F \in \mathfrak{B}(X, Z)$.

(3) Let $F \in \mathfrak{K C}(X, Z)$ be closed. For any $N \in\langle D\rangle$ with the cardinality $|N|=n+1$, the simplex $\left(\Delta_{n}, V\right.$; co $)$, where $V$ is the set of vertices, is a $G$ convex space. In view of condition $(*),\left(X^{\prime}, D^{\prime} ; \Gamma^{\prime}\right):=\left(\phi_{N}\left(\Delta_{n}\right), \phi_{N}(V) ; \Gamma^{\prime}\right)$ is a $G$-convex subspace of $(X \supset D ; \Gamma)$, where $\Gamma^{\prime}\left(\phi_{N}(A)\right):=\Gamma\left(\phi_{N}(A)\right)=\phi_{N}(\operatorname{co} A)$ for each $A \subset V$. Let $H: V \multimap Z$ be a closed-valued map satisfying

$$
F \phi_{N}\left(\Delta_{J}\right) \subset H(J) \text { for each } J \in\langle V\rangle \text {. }
$$

Define $G: D^{\prime} \multimap Z$ by $G\left(\phi_{N}(v)\right):=H(v)$ for $v \in V$. Then

$$
\left.F\right|_{X^{\prime}}\left(\Gamma_{B}^{\prime}\right) \subset G(B) \text { for each } B \in\left\langle D^{\prime}\right\rangle \text {. }
$$


Since $\left.F\right|_{X^{\prime}} \in \mathfrak{K C}\left(X^{\prime}, Z\right)$ by Lemma $2,\{G(y)\}_{y \in D^{\prime}}$ has the finite intersection property, and hence so does $\{H(v)\}_{v \in V}$. Therefore, $F \phi_{N} \in \mathfrak{K C}\left(\Delta_{n}, Z\right)$. Since $F$ is closed, by (2), we have $F \in \mathfrak{B}(X, Z)$.

Remark. In (2), note that for any map $F \in \mathfrak{A}_{c}^{\kappa}(X, Z)$, we have $F \phi_{N} \in$ $\mathfrak{A}_{c}^{\kappa}\left(\Delta_{n}, Z\right) \subset \mathfrak{K C}\left(\Delta_{n}, Z\right) \cap \mathfrak{K O}\left(\Delta_{n}, Z\right)$ when $Z$ is Hausdorff; see [24].

Corollary 16.1. In the class of compact closed maps defined on a $G$-convex space $(X \supset D ; \Gamma)$ satisfying condition $(*)$ into a Hausdorff space $Z$, two subclasses $\mathfrak{K C}(X, Z)$ and $\mathfrak{B}(X, Z)$ are identical.

Corollary 16.2. In the class of compact closed maps defined on a convex space $(X, D)$ into a Hausdorff space $Z$, two subclasses $\mathfrak{K C}(X, Z)$ and $\mathfrak{B}(X, Z)$ are identical.

Remark. This is noted in [33] by a different method. In view of Corollary 16.2, the class $\mathfrak{B}$ is favorable to use for convex spaces since it has already plenty of examples and is much easier to find examples.

From Theorems 13 and 16, we have the following:

Theorem 17. Let $(X \supset D ; \Gamma ; \mathcal{U})$ be a $G$-convex separated uniform space satisfying condition $(*)$, and $F \in \mathfrak{K C}(X, X)$ a compact closed map such that $F(X)$ is Klee approximable. Then $F$ has a fixed point.

Consequently, all of the known fixed point theorems on the S-KKM class are consequences of Theorem 17.

Corollary 17.1. Let $X$ be an admissible almost convex subset of a Hausdorff topological vector space, and $F \in \mathfrak{K C}(X, X)$ a compact closed map. Then $F$ has a fixed point.

This is first stated for a convex set and encompasses most of known fixed point results on KKM or S-KKM classes; see [35, 42].

\section{Variational inequalities and best approximations}

Recall that an extended real valued function $f: X \rightarrow \overline{\mathbb{R}}$, where $X$ is a topological space, is lower [resp. upper] semicontinuous (l.s.c.) [resp. u.s.c.] if $\{x \in X: f(x)>r\}$ [resp. $\{x \in X \mid f(x)<r\}]$ is open for each $r \in \overline{\mathbb{R}}$.

For an abstract convex space $(X ; \Gamma)$, a real function $f: X \rightarrow \overline{\mathbb{R}}$ is said to be quasiconcave [resp. quasiconvex] if $\{x \in X \mid f(x)>r\}$ [resp. $\{x \in X \mid f(x)<$ $r\}]$ is $\Gamma$-convex for each $r \in \overline{\mathbb{R}}$.

An abstract convex space $(X ; \Gamma)$ is said to be compact if $X$ is a compact topological space. From now on, for simplicity, we are mainly concerned with compact abstract convex spaces $(X ; \Gamma)$ such that $1_{X} \in \mathfrak{K C}(X, X)$. For example, any compact $G$-convex space, any compact $H$-space, or any compact convex space is such a space.

We begin with a particular form of the minimax theorem 7 : 
Theorem 18. Let $(X ; \Gamma)$ be a compact abstract convex space such that $1_{X} \in$ $\mathfrak{K C}(X, X)$ and $f, g: X \times X \rightarrow \mathbb{R} \cup\{+\infty\}$ be functions such that

(18.1) $f(x, y) \leq g(x, y)$ for each $(x, y) \in X \times X$,

(18.2) for each $x \in X, g(x, \cdot)$ is quasiconcave on $X$; and

(18.3) for each $y \in X, f(\cdot, y)$ is l.s.c. on $X$.

Then we have

$$
\min _{y \in X} \sup _{x \in X} f(x, y) \leq \sup _{x \in X} g(x, x) .
$$

Proof. Observe that $\sup _{x \in X} f(x, y)$ is by (18.3) a l.s.c. function of $y$ on the compact space $X$, and therefore its minimum exists. If $\sup _{x \in X} g(x, x)=$ $+\infty$, then the inequality in the conclusion holds automatically. If $\alpha=\beta=$ $\sup _{x \in X} g(x, x)<+\infty$, then by Theorem 7 with $F=1_{X}$, we have the conclusion.

Remarks. 1. For $f=g$, Theorem 18 reduces to Fan's minimax inequality [15]. Fan obtained his inequality from his own generalization of the KKM principle, and applied it to deduce fixed point theorems, theorems on sets with convex sections, a fundamental existence theorem in potential theory, and so on.

2. Later, the inequality has been an important tool in nonlinear analysis, game theory, and economic theory; see [35, 36].

In particular, we have the following:

Corollary 18.1. Under the hypothesis of Theorem 18, if $g(x, x) \leq 0$ for all $x \in X$, then there exists a $y_{0} \in X$ such that $f\left(x, y_{0}\right) \leq 0$ for all $x \in X$. Thus in particular

$$
\min _{y \in X} \sup _{x \in X} f(x, y) \leq 0 .
$$

Theorem 18 can be applied to the existence of solutions of certain variational inequalities:

Theorem 19. Let $(X ; \Gamma)$ be a compact abstract convex space such that $1_{X} \in$ $\mathfrak{K C}(X, X)$ and $p, q: X \times X \rightarrow \mathbb{R}$ and $h: X \rightarrow \mathbb{R}$ functions satisfying

(19.1) $p(x, y) \leq q(x, y)$ for each $(x, y) \in X \times X$, and $q(x, x) \leq 0$ for all $x \in X$;

(19.2) for each $x \in X, q(x, \cdot)+h(\cdot)$ is quasiconcave on $X$; and

(19.3) for each $y \in X, p(\cdot, y)-h(\cdot)$ is l.s.c. on $X$.

Then there exists a $y_{0} \in X$ such that

$$
p\left(x, y_{0}\right)+h\left(y_{0}\right) \leq h(x) \text { for all } x \in X .
$$

Proof. Let

$$
f(x, y):=p(x, y)+h(y)-h(x), g(x, y):=q(x, y)+h(y)-h(x)
$$

for $(x, y) \in X \times Y$. Then $f$ and $g$ satisfy the requirements of Theorem 18 . Furthermore, $g(x, x)=q(x, x) \leq 0$ for all $x \in X$. Therefore, by Corollary 18.1, the conclusion follows. 
Remarks. 1. Putting $h=0$, Theorem 19 reduces to Corollary 18.1.

2. Theorem 19 is a basis of existence theorems of many results concerning variational inequalities; see [36] and references therein.

Theorem 20. Let $(X ; \Gamma)$ be a compact abstract convex space such that $1_{X} \in$ $\mathfrak{K C}(X, X)$ and $p, q: X \times X \rightarrow \mathbb{R}$ functions such that

(20.1) $p \leq q$ on the diagonal $\Delta:=\{(x, x) \mid x \in X\}$ and $q \leq p$ on $(X \times X) \backslash \Delta$;

(20.2) for each $x \in X, y \mapsto q(y, y)-q(x, y)$ is quasiconcave on $X$; and

(20.3) for each $y \in X, x \mapsto p(x, y)$ is u.s.c. on $X$.

Then there exists a $y_{0} \in X$ such that

$$
p\left(y_{0}, y_{0}\right) \leq p\left(x, y_{0}\right) \quad \text { for all } x \in X .
$$

Proof. Define $f, g: X \times X \rightarrow \mathbb{R}$ by

$$
f(x, y):=p(y, y)-p(x, y), g(x, y):=q(y, y)-q(x, y)
$$

Then $f$ and $g$ satisfy the hypothesis of Theorem 18. Since $g(x, x)=0$ for all $x \in X$, Corollary 18.1 implies that $f\left(x, y_{0}\right) \leq 0$ for all $x \in X$. This implies the conclusion.

Remark. For a convex space $X$ and $p=q$, Theorem 20 reduces to Fan [15], which was shown to be very useful in nonlinear functional analysis. In fact, the Tychonoff (and hence, the Brouwer) fixed point theorem, Browder's variational inequality, and many other applications follow from his result.

A simple consequence of Theorem 20 is the following well-known existence result on best approximations originated from Ky Fan [14]:

Corollary 20.1. Let $X$ be a compact convex subset of a topological vector space $E$ and $f: X \rightarrow E$ a continuous function. Then for any continuous seminorm $p$ on $E$, there exists a point $y_{0} \in X$ such that

$$
p\left(y_{0}-f\left(y_{0}\right)\right) \leq p\left(x-f\left(y_{0}\right)\right) \quad \text { for all } x \in X .
$$

Proof. For each $y \in X, x \mapsto p(y-f(y))-p(x-f(y))$ is convex on $X$, and for each $x \in X, y \mapsto p(x-f(y))$ is continuous. Therefore, by Theorem 20, we have a $y_{0} \in X$ satisfying the conclusion.

Remark. Further if $E$ is a normed vector space and $p$ is a norm on $E$, then Corollary 20.1 reduces to the well-known existence result on best approximation due to Ky Fan [14, Theorem 2], which immediately implies the Schauder fixed point theorem; that is, the normed space version of the Brouwer theorem. Therefore, Corollary 20.1 generalizes and implies the Brouwer theorem.

\section{The von Neumann type minimax theorem}

We begin this section with that the product of any family of abstract convex spaces is also an abstract convex space. In fact, we clearly have the following: 
Lemma 8. Let $\left\{\left(E_{i}, D_{i} ; \Gamma_{i}\right)\right\}_{i \in I}$ be any family of abstract convex spaces. Let $E:=\prod_{i \in I} E_{i}$ and $D:=\prod_{i \in I} D_{i}$. For each $i \in I$, let $\pi_{i}: D \rightarrow D_{i}$ be the projection. For each $A \in\langle D\rangle$, define $\Gamma(A):=\prod_{i \in I} \Gamma_{i}\left(\pi_{i}(A)\right)$. Then $(E, D ; \Gamma)$ is an abstract convex space.

Note also that for the case $E_{i}=D_{i}$ for each $i$, the product of abstract convex subsets is also abstract convex in the product abstract convex space.

In this section, we show that a typical classical application of the KKM theorem can be extended to abstract convex spaces.

As a direct application of Theorem 11, we have the following generalization of the von Neumann-Sion minimax theorem [48, 50]:

Theorem 21. Let $(X ; \Gamma)$ and $\left(Y ; \Gamma^{\prime}\right)$ be compact abstract convex spaces and $f, g: X \times Y \rightarrow \mathbb{R} \cup\{+\infty\}$ be functions such that

(21.1) $f(x, y) \leq g(x, y)$ for each $(x, y) \in X \times Y$;

(21.2) for each $x \in X, f(x, \cdot)$ is l.s.c. and $g(x, \cdot)$ is quasiconvex on $Y$; and

(21.3) for each $y \in Y, f(\cdot, y)$ is quasiconcave and $g(\cdot, y)$ is u.s.c. on $X$. If $1_{X \times Y} \in \mathfrak{K C}(X \times Y, X \times Y)$, then we have

$$
\min _{y \in Y} \sup _{x \in X} f(x, y) \leq \max _{x \in X} \inf _{y \in Y} g(x, y) .
$$

Proof. Note that $y \mapsto \sup _{x \in X} f(x, y)$ is l.s.c. on $Y$ and $x \mapsto \inf _{y \in Y} g(x, y)$ is u.s.c. on $X$. Therefore, the both sides of the inequality exist. Suppose that there exists a real $c$ such that

$$
\max _{x} \inf _{y} g(x, y)<c<\min _{y} \sup _{x} f(x, y) .
$$

Let $\Gamma_{X \times Y}$ be the product convexity defined as in Lemma 8 . Then $(X \times$ $\left.Y ; \Gamma_{X \times Y}\right)$ is a compact abstract convex space. Define a map $T: X \times Y \rightarrow$ $X \times Y$ by

$$
T(x, y)=\{\bar{x} \in X \mid f(\bar{x}, y)>c\} \times\{\bar{y} \in Y \mid g(x, \bar{y})<c\}
$$

for $(x, y) \in X \times Y$. Then $T(x, y)$ is nonempty and $\Gamma$-convex for each $(x, y) \in$ $X \times Y$ and $T^{-}(x, y)$ is open. By using Theorem 11, we have an $\left(x_{0}, y_{0}\right) \in X \times Y$ such that $\left(x_{0}, y_{0}\right) \in T\left(x_{0}, y_{0}\right)$. Therefore, $c<f\left(x_{0}, y_{0}\right) \leq g\left(x_{0}, y_{0}\right)<c$, a contradiction.

Remark. If $f=g$ and if $X$ is a convex space, Theorem 21 reduces to Sion's generalization [48] of the von Neumann minimax theorem [50]:

$$
\min _{x} \max _{y} f(x, y)=\max _{y} \min _{x} f(x, y) \text {. }
$$

\section{The Nash type equilibrium theorem}

In this section, from a Fan-Browder type fixed point result for abstract convex spaces, we deduce the Ky Fan intersection theorem and the Nash equilibrium theorem for abstract convex spaces. 
Given a cartesian product $X=\prod_{i=1}^{n} X_{i}$ of sets, let $X^{i}=\prod_{j \neq i} X_{j}$ and $\pi_{i}: X \rightarrow X_{i}, \pi^{i}: X \rightarrow X^{i}$ be the projections; we write $\pi_{i}(x)=x_{i}$ and $\pi^{i}(x)=x^{i}$. Given $x, y \in X$, we let

$$
\left(y_{i}, x^{i}\right):=\left(x_{1}, \ldots, x_{i-1}, y_{i}, x_{i+1}, \ldots, x_{n}\right) .
$$

From Theorem 11, we have the following Ky Fan type intersection theorem [13] generalizing the well-known von Neumann intersection lemma [51]:

Theorem 22. Let $X=\prod_{i=1}^{n} X_{i},(X ; \Gamma)$ be a compact abstract convex space, and $A_{1}, A_{2}, \ldots, A_{n} n$ subsets of $X$ such that

(22.1) for each $x \in X$ and $i=1, \ldots, n$, the set $A_{i}(x)=\left\{y \in X \mid\left(y_{i}, x^{i}\right) \in\right.$ $\left.A_{i}\right\}$ is $\Gamma$-convex and nonempty; and

(22.2) for each $y \in X$ and $i=1, \ldots, n$, the set $A_{i}(y)=\left\{x \in X \mid\left(y_{i}, x^{i}\right) \in\right.$ $\left.A_{i}\right\}$ is open.

If $1_{X} \in \mathfrak{K C}(X, X)$, then $\bigcap_{i=1}^{n} A_{i} \neq \emptyset$.

Proof. Define a map $T: X \multimap X$ by $T(x):=\bigcap_{i=1}^{n} A_{i}(x)$ for $x \in X$. Then each $T(x)$ is $\Gamma$-convex being an intersection of $\Gamma$-convex sets by (22.1). For each $x \in X$ and each $i$, there exists a $y^{(i)} \in A_{i}(x)$ by $(22.1)$, or $\left(y_{i}^{(i)}, x^{i}\right) \in A_{i}$. Hence, we have $\left(y_{1}^{(1)}, \ldots, y_{n}^{(n)}\right) \in \bigcap_{i=1}^{n} A_{i}(x)$. This shows $T(x) \neq \emptyset$. Moreover, $T^{-}(y)=\bigcap_{i=1}^{n} A_{i}(y)$ is open for each $y \in X$ by (22.2). Now, the conclusion follows from Theorem 11.

Remark. If each $X_{i}$ is a compact abstract convex space, so is $X$ with the product topology; see Lemma 8 . Note that Theorem 21 can be also deduced from Theorem 22; see [36].

From Theorem 22, we deduce the following Nash equilibrium theorem [27] for abstract convex spaces:

Theorem 23. Let $X=\prod_{i=1}^{n} X_{i},(X ; \Gamma)$ a compact abstract convex space, and $f_{1}, \ldots, f_{n}: X \rightarrow \mathbb{R}$ continuous functions such that

(23.1) for each $x \in X$, each $i=1, \ldots, n$, and each $r \in \mathbb{R}$, the set $\left\{\left(y_{i}, x^{i}\right) \in\right.$ $\left.X \mid f_{i}\left(y_{i}, x^{i}\right)>r\right\}$ is $\Gamma$-convex.

If $1_{X} \in \mathfrak{K C}(X, X)$, then there exists a point $x \in X$ such that

$$
f_{i}(x)=\max _{y_{i} \in X_{i}} f_{i}\left(y_{i}, x^{i}\right) \quad \text { for } \quad i=1, \ldots, n .
$$

Proof. Let $\varepsilon>0$ and, for each $i$, let

$$
A_{i}^{\varepsilon}=\left\{x \in X \mid f_{i}(x)>\max _{y_{i} \in X_{i}} f_{i}\left(y_{i}, x^{i}\right)-\varepsilon\right\} .
$$

Then the sets $A_{1}^{\varepsilon}, \ldots, A_{n}^{\varepsilon}$ satisfy conditions (22.1) and (22.2) of Theorem 22, and hence $\bigcap_{i=1}^{n} A_{i}^{\varepsilon} \neq \emptyset$. Then $H_{\varepsilon}=\bigcap_{i=1}^{n} \overline{A_{i}^{\varepsilon}}$ is a nonempty compact set. Since $H_{\varepsilon_{1}} \subset H_{\varepsilon_{2}}$ for $\varepsilon_{1}<\varepsilon_{2}$, we have $\bigcap_{\varepsilon>0} H_{\varepsilon} \neq \emptyset$. Then $x \in \bigcap_{\varepsilon>0} H_{\varepsilon}$ satisfies the conclusion. 
Final Remarks. This is not the end of the story. Since there are several hundred published works on the KKM theory, we can cover only an essential part of the theory. For the more historical background, the reader can consult with [35]. For more involved or particular versions of the results in this paper, see [31-33] for convex spaces, [45] for $H$-spaces, and [36-42, 44, 46, 47] for $G$-convex spaces and references therein.

\section{References}

[1] P. S. Alexandroff, Combinatorial Topology, OGIZ, Moscow-Leningrad, 1947. [Russian]

[2] P. Alexandroff and H. Hopf, Topologie I, Springer, Berlin-Heidelberg-New York, 1974.

[3] P. Alexandroff and B. Pasynkoff, Elementary proof of the essentiality of the identical mapping of a simplex, Uspehi Mat. Nauk (N.S.) 12 (1957), no. 5 (77), 175-179. [Russian]

[4] A. Amini, M. Fakhar, and J. Zafarani, Fixed point theorems for the class S-KKM mappings in abstract convex spaces, Nonlinear Anal. 66 (2006), 14-21.

[5] H. Ben-El-Mechaiekh, P. Deguire, and A. Granas, Points fixes et coincidences pour les applications multivoques (applications de Ky Fan), C. R. Acad. Sci. Paris Sér. I Math. 295 (1982), no. 4, 337-340.

[6] ___ Points fixes et coincidences pour les fonctions multivoques II (applications de type $\varphi$ et $\varphi^{*}$ ), C. R. Acad. Sci. Paris 295 (1982), no. 5, 381-384.

[7] A. Borglin and H. Keiding, Existence of equilibrium actions and of equilibrium, J. Math. Econom. 3 (1976), no. 3, 313-316.

[8] F. E. Browder, The fixed point theory of multi-valued mappings in topological vector spaces, Math. Ann. 177 (1968), 283-301.

[9] T.-H. Chang and C.-L. Yen, KKM property and fixed point theorems, J. Math. Anal. Appl. 203 (1996), no. 1, 224-235.

[10] T.-H. Chang, Y.-Y. Huang, J.-C. Jeng, and K.-H. Kuo, On S-KKM property and related topics, J. Math. Anal. Appl. 229 (1999), no. 1, 212-227.

[11] K. Fan, A generalization of Tychonoff's fixed point theorem, Math. Ann. 142 (1961), 305-310.

[12] __ Sur un théorème minimax, C. R. Acad. Sci. Paris 259 (1964), 3925-3928.

[13] _ Applications of a theorem concerning sets with convex sections, Math. Ann. 163 (1966), 189-203.

[14] Extensions of two fixed point theorems of F. E. Browder, Math. Z. 112 (1969), 234-240.

[15] _ A minimax inequality and applications, Inequalities III (O. Shisha, ed.), 103113, Academic Press, New York, 1972.

[16] _ A further generalization of Shapley's generalization of the Knaster-Kuratowski-Mazurkiewicz theorem, Game Theory and Mathematical Economics (O. Moeschlin and D. Palaschke, ed.), 275-279, North-Holland, Amsterdam, 1981.

[17] _ Some properties of convex sets related to fixed point theorems, Math. Ann. 266 (1984), no. 4, 519-537.

[18] C. J. Himmelberg, Fixed points of compact multifunctions, J. Math. Anal. Appl. 38 (1972), 205-207.

[19] C. D. Horvath, Some results on multivalued mappings and inequalities without convexity, Nonlinear and Convex Analysis-- Proc. in honor of Ky Fan (B. L. Lin and S. Simons, eds.), 99-106, Marcel Dekker, New York, 1987.

[20] — Convexité généralisée et applications, Sém. Math. Supér. 110, 79-99, Press. Univ. Montréal, 1990.

[21] __ Contractibility and generalized convexity, J. Math. Anal. Appl. 156 (1991), no. $2,341-357$. 
[22] _ Extension and selection theorems in topological spaces with a generalized convexity structure, Ann. Fac. Sci. Toulouse Math. (6) 2 (1993), no. 2, 253-269.

[23] H. Kim, KKM property, S-KKM property and fixed point theorems, Nonlinear Anal. 63 (2005), e1877-e1884.

[24] H. Kim and S. Park, Remarks on the KKM property for open-valued multimaps on generalized convex spaces, J. Korean Math. Soc. 42 (2005), no. 1, 101-110.

[25] B. Knaster, K. Kuratowski, and S. Mazurkiewicz, Ein Beweis des Fixpunktsatzes für n-Dimensionale Simplexe, Fund. Math. 14 (1929), 132-137.

[26] M. Lassonde, On the use of KKM multifunctions in fixed point theory and related topics, J. Math. Anal. Appl. 97 (1983), no. 1, 151-201.

[27] J. Nash, Non-cooperative games, Ann. of Math. (2) 54 (1951), 286-293.

[28] S. Park, Generalizations of Ky Fan's matching theorems and their applications, J. Math. Anal. Appl. 141 (1989), no. 1, 164-176.

[29] _ Generalized matching theorems for closed coverings of convex sets, Numer. Funct. Anal. Optim. 11 (1990), no. 1-2, 101-110.

[30] - Some coincidence theorems on acyclic multifunctions and applications to $K K M$ theory, Fixed Point Theory and Applications (K.-K. Tan, ed.), 248-277, World Sci. Publ., River Edge, NJ, 1992.

[31] - Foundations of the KKM theory via coincidences of composites of upper semicontinuous maps, J. Korean Math. Soc. 31 (1994), no. 3, 493-519.

[32] _ A unified approach to generalizations of the KKM-type theorems related to acyclic maps, Numer. Funct. Anal. Optim. 15 (1994), no. 1-2, 105-119.

[33] _ Coincidence theorems for the better admissible multimaps and their applications, Nonlinear Anal. 30 (1997), no. 7, 4183-4191.

[34] _ A unified fixed point theory of multimaps on topological vector spaces, J. Korean Math. Soc. 35 (1998), no. 4, 803-829; Corrections, ibid. 36 (1999), no. 4, 829-832.

[35] _ Ninety years of the Brouwer fixed point theorem, Vetnam J. Math. 27 (1999), no. $3,187-222$.

[36] _ Elements of the KKM theory for generalized convex spaces, Korean J. Comp. Appl. Math. 7 (2000), no. 1, 1-28.

[37] - Remarks on topologies of generalized convex spaces, Nonlinear Funct. Anal. Appl. 5 (2000), no. 2, 67-79.

[38] , New topological versions of the Fan-Browder fixed point theorem, Nonlinear Anal. 47 (2001), no. 1, 595-606.

[39] __ Fixed point theorems in locally G-convex spaces, Nonlinear Anal. 48 (2002), no. 6, Ser. A: theory Methods, 869-879.

[40] - Coincidence, almost fixed point, and minimax theorems on generalized convex spaces, J. Nonlinear Convex Anal. 4 (2003), no. 1, 151-164.

[41] On generalizations of the KKM principle on abstract convex spaces, Nonlinear Anal. Forum 11 (2006), no. 1, 67-77.

[42] — A unified fixed point theory in generalized convex spaces, Acta Math. Sinica, English Series 23 (2007), no. 8, 1509-1536.

[43] S. Park and K. S. Jeong, Fixed point and non-retract theorems - Classical circular tours, Taiwanese J. Math. 5 (2001), no. 1, 97-108.

[44] S. Park and H. Kim, Admissible classes of multifunctions on generalized convex spaces, Proc. Coll. Natur. Sci., Seoul Nat. Univ. 18 (1993), 1-21.

[45] _- Coincidences of composites of u.s.c. maps on $H$-spaces and applications, $\mathrm{J}$ Korean Math. Soc. 32 (1995), no. 2, 251-264.

[46] - Coincidence theorems for admissible multifunctions on generalized convex spaces, J. Math. Anal. Appl. 197 (1996), no. 1, 173-187.

[47] - Foundations of the KKM theory on generalized convex spaces, J. Math. Anal. Appl. 209 (1997), no. 2, 551-571. 
[48] M. Sion, On general minimax theorems, Pacific J. Math. 8 (1958), 171-176.

[49] B. P. Sortan, Introduction to Axiomatic Theory of Convexity, Kishynef, 1984. [Russian with English summary.]

[50] J. von Neumann, Zur Theorie der Gesellschaftsspiele, Math. Ann. 100 (1928), no. I, 295320 .

[51] — Über ein ökonomisches Cleichungssystem und eine Verallgemeinerung des Brouwerschen Fixpunktsatzes, Ergeb. Math. Kolloq. 8 (1937), 73-83.

[52] N. Yannelis and N. D. Prabhakar, Existence of maximal elements and equilibria in linear topological spaces, J. Math. Econom. 12 (1983), no. 3, 233-245.

The National Academy of Schences. Republic of Konea;

DEPARTMENT OF MATHEMATICAL SCIENCES

SEOUl National UNIVERSITY

SEOUL 151-747, KOREA.

E-mail address: shpark@math.snu.ac.kr 